Our Nature (2003) 1: 37-41

\title{
An Ethnobiological Study of the Tamang People
}

\author{
Ganesh Tamang* \\ Central Department of Zoology, Kirtipur
}

\begin{abstract}
Tamangs are one of the major ethnic groups of Nepal. Ethnobiological investigation of Tamang people of Gorsyang Village Development Committee of Nuwakot district was carried out. Information was documented from structured questionnaire and interviews with local people. They were found to have rich indigenous knowledge. They use different 12 animal names as calendar. A total of 11 animal species and 44 plant species were found to be used in medicinal purpose. Viscera of Hystrix brachyura, pancreas of fish and flesh of Rana tigrina were found using in the treatment of asthma, jaundice and pneumonia. The fur of Lepus nigricollis is used to stop bleeding. Acorus calamus, Centilla asiatica and Terminalia chebula are the important medicinal plants, which they use to control throat, urine and gastric problems. The stem extract of Tinospora cordifolia is used in menstruation problems.
\end{abstract}

\section{Introduction}

Tamangs are one of the major TibetoBurmese speaking communities in Nepal. They believe that they originally came from Tibet. The entire community of Tamang is divided into several sub castes known as 'thar'. Each 'thar' has its own name like Sangden, Bomjan, Yonjan, Pakhrin, etc. Languagewise, these people are the third largest ethnic group in the kingdom, but if assessed from the angle of their being in the Tibeto-Burmese category, they would be the most populous.

In Nepal more than 61 ethnic groups are dispersed all over the countries. Tamang people account 5.5 per cent of the total population of the country (Anonymous 2000). Majority lives in the hilly regions of Nepal, adjoining sides of Kathmandu valley, the capital of Nepal. The historical and legendary account of the Tamangs show that they originally came from the Tibet. It is

* Correspondence: Central Campus of Technology, Dharan. E-mail: syangdeng@yahoo.com said that they were horse traders. "Ta" in Tibetan means horse; "Mang" means traders (Bista 1967). They have very rich ethnobiological knowledge.

Their social, cultural, economic and religious practices are, in one way or other, linked to plants and animals. For example, in the 'Loh' (age calculation calender), twelve different animals have been used. Each Loh always starts from 'Magh Suklapratipadha' and continue till a year. The animals associated with the twelve Lohs are: Chi Loh (Mouse), Lung Loh (Cow), Ta Loh (Tiger), Yai Loh (Goat), Prub Loh (Dragon), Prul Loh (Serpent), Tak Loh (Horse), Look Loh (Sheep), Pray Loh (Monkey), Chyu Loh (Bird), Khi Loh (Dog), and Pha Loh (Boar) (Tamang 1998).

So, within Nepal, there is remarkable ethnic and biological diversity and wealth of indigenous knowledge of plants and animals with economic value. A few ethnobotanical studies of Tamang people have been carried out (Shrestha 1988, Shrestha 1989, Manandhar 1991, Taylor et 
Our Nature (2003) 1: 37-41

al. 1996) but record on ethnobiology is not available.

The study area lies in Gorsyang VDC of Nuwakot district $\left(27^{0} 48^{\prime}\right.$ to $28^{0} 6^{\prime}$ latitude and $84^{\circ} 58^{\prime}$ to $85^{\circ} 30^{\prime}$ longitude) of Bagmati zone in the hilly region of central Nepal. The VDC covers 33.03 sq.km at elevation of 900- $2738 \mathrm{~m}$, where Tamange people have densely inhabited.

\section{Materials and Methods}

The research was carried out from May 1997 to Feb 1998. It was entirely based on both primary and secondary data. To obtain information, structured questionnaire was designed. Interviews were conducted with the Tamang people of the village, the Lamas (the tribal priest), local healers so as to know their ethnography as well as utilization of medicinal plants and animals. All the specimens consumed for medicinal purpose by the Tamang people of Gorsyang VDC were collected and identified with the available literature (Bangdel and Fleming 1984, Shrestha 1994, Anonymous 1994, Shrestha 1997, Polunin and Stainton 1997) and for further confirmation specimens were tallied in the Department of Forestry and Plant Research, Godawari (KATH); National History Museum, Swayambu; Botanical Department, Thapathali and Museum of Central Department of Zoology. Altogether 9 species of animals (both invertebrate and vertebrate) and 44 important medicinal plants were collected and deposited at Central Department of Zoology, Kirtipur and the Department of Forestry and Plant Research, Kathmandu.

\section{Results and Discussion}

Many people were found to lack a formal grade school education, but were highly knowledgeable about uses of local animals and plants (Tables 1 and 2). The two mammalian species Hystrix brachyura and Lepus nigricollis were recorded to have medicinal use. The viscera of Hystrix brachyura, fish and flesh of Rana tigrina were found to cure asthma, jaundice and pneumonia. The fur of Lepus nigricollis was used to stop bleeding. Apis cerena and Apis dorsata were found to be used in cold and cough treatment. Egg of Vanellus Indicus was found to cure asthma and flatulence. The flesh of Rana tigrina was found to cure asthma and pneumonia. Beside this, forty-two plant species were found to have medicinal use. Acorus calamus, Centilla asiatica, Terminalia chebula are their important medicinal plants to control throat, urine and gastric problems. The stem extract of Tinospora cordifolia is used in menstruations problem.

From the research it shows that Tamangs have a rich folklore and indigenous knowledge in the utilization of different plants and animals against various common diseases like asthma, pneumonia, jaundice, stomach problem, worms, minor cut, diarrhoea etc. But such folklore is passed on orally from generation to generation without keeping any written records. Therefore, the lack of written document on the plants and animals lore reveals the chances of losing the valuable tradition forever. In view of this aspect, it is necessary to preserve and properly document to keep a record of the diversified utilization of various plant and animal species. 
Our Nature (2003) 1: 37-41

Table 1. The recorded animal species used by Tamang people for medicinal purpose.

\begin{tabular}{|l|l|l|l|l|l|}
\hline \multicolumn{1}{|c|}{ ANIMAL SPECIES } & \multicolumn{1}{|c|}{$\begin{array}{c}\text { Species } \\
\text { Type }\end{array}$} & \multicolumn{1}{|c|}{ Family } & $\begin{array}{c}\text { Tamang } \\
\text { name }\end{array}$ & \multicolumn{1}{|c|}{ Part Used } & \multicolumn{1}{|c|}{ Medicinal Use } \\
\hline Apis cerana & Invertebrate & Apidae & Sing shore & Whole insect & $\begin{array}{l}\text { Cough and cold, } \\
\text { tonic }\end{array}$ \\
\hline Apis dorsata & Invertebrate & Apidae & Wai & Whole insect & $\begin{array}{l}\text { Cough and cold, } \\
\text { tonic }\end{array}$ \\
\hline Barilius bendelises & Fish & Cyprinidae & Tarnga & Pancreas & $\begin{array}{l}\text { To kill worms, } \\
\text { asthma }\end{array}$ \\
\hline Chana gachua & Fish & & Hile & Pancreas & $\begin{array}{l}\text { To kill worms, } \\
\text { asthma }\end{array}$ \\
\hline Garra gotyla & Fish & Cyprinidae & Buduna & Pancreas & $\begin{array}{l}\text { To kill worms, } \\
\text { asthma }\end{array}$ \\
\hline Hystris brachyura & Mammals & Hystricidae & dumsi & Viscera & Asthma \\
\hline Lepus nigricollis ruficaudatus & Mammals & Lagomorpha & Kharayo & Fur & To stop bleeding \\
\hline Noemacheilus beavani & Fish & Cobitedae & Brudhung & Pancrease & $\begin{array}{l}\text { To kill worms, } \\
\text { asthma }\end{array}$ \\
\hline Rana tigrina & Amphibia & Ranidae & kalang tek tek & Flesh & Asthma, pneumonia \\
\hline Tor tor & Fish & Cyprinidae & Katle & Pancrease & $\begin{array}{l}\text { To kill worms, } \\
\text { asthma }\end{array}$ \\
\hline Vanellus Indicus & Birds & Charadridae & tedebuth & Egg & Asthma, flatulence \\
\hline
\end{tabular}

Table 2. Plants species used by Tamang people for medicinal purpose are:

\begin{tabular}{|l|l|l|l|l|l|l|}
\hline \multicolumn{1}{|c|}{ PLANTS SPECIES } & FAMILY & $\begin{array}{l}\text { TAMANG } \\
\text { NAME }\end{array}$ & $\begin{array}{l}\text { NEPALI } \\
\text { NAME }\end{array}$ & $\begin{array}{l}\text { ENGLISH } \\
\text { NAME }\end{array}$ & $\begin{array}{l}\text { PART } \\
\text { USED }\end{array}$ & \multicolumn{1}{|c|}{ USES } \\
\hline Acorus calamus L & Araceae & Bhojho & Bhojho & - & Stem & $\begin{array}{l}\text { Cough, } \\
\text { bronchitis }\end{array}$ \\
\hline $\begin{array}{l}\text { Aegle marmelos (L) } \\
\text { Correa }\end{array}$ & Rutaceae & Bel & Bel & $\begin{array}{l}\text { Bengal } \\
\text { quince }\end{array}$ & Fruit & $\begin{array}{l}\text { Diarrhoea, } \\
\text { dysentery, tonic }\end{array}$ \\
\hline Agave Americana Linn & Amaryllidaceae & Ketuke & Ketuke & $\begin{array}{l}\text { Century } \\
\text { plant }\end{array}$ & $\begin{array}{l}\text { Leaf } \\
\text { extract }\end{array}$ & $\begin{array}{l}\text { Diuretic, } \\
\text { antisyphilitic }\end{array}$ \\
\hline Ageratum conyzoides L. & Asteraceae & Pokomra & $\begin{array}{l}\text { Gandhe } \\
\text { Jhar }\end{array}$ & - & $\begin{array}{l}\text { Leaf } \\
\text { extract }\end{array}$ & $\begin{array}{l}\text { Cuts and } \\
\text { wounds }\end{array}$ \\
\hline Allium sativum L & Liliaceae & No & Lasun & Garlic & Stem & $\begin{array}{l}\text { Flatulence,skin } \\
\text { rashes }\end{array}$ \\
\hline Aloevera (L) Brum F. & Liliaceae & Ghiu Kumari & $\begin{array}{l}\text { Ghiu } \\
\text { Kumari }\end{array}$ & $\begin{array}{l}\text { Indian } \\
\text { aloe }\end{array}$ & $\begin{array}{l}\text { Plant } \\
\text { extract }\end{array}$ & Burn \\
\hline Artemisia vulgaris Linn & ”, & Cendeth & Titepati & Mag-wort & $\begin{array}{l}\text { Root and } \\
\text { leaf }\end{array}$ & $\begin{array}{l}\text { Nausea } \\
\text { problem, } \\
\text { intestinal } \\
\text { worms }\end{array}$ \\
\hline $\begin{array}{l}\text { Azadirachta indica A } \\
\text { Juss }\end{array}$ & Meliaceae & Neem & Neem & Neem tree & $\begin{array}{l}\text { Leaf } \\
\text { extract }\end{array}$ & $\begin{array}{l}\text { Fever, intestinal } \\
\text { worms }\end{array}$ \\
\hline Bauhinia variegata L & Fabaceae & $\begin{array}{l}\text { Koiralo } \\
\text { Mendo }\end{array}$ & Koiralo & $\begin{array}{l}\text { Pink } \\
\text { bauhinia }\end{array}$ & $\begin{array}{l}\text { Flower, } \\
\text { seed }\end{array}$ & $\begin{array}{l}\text { Dysentery, } \\
\text { piles, diarrhoea, }\end{array}$ \\
\hline
\end{tabular}


Our Nature (2003) 1: 37-41

\begin{tabular}{|c|c|c|c|c|c|c|}
\hline & & & & & & worms \\
\hline Berberis aristata DC. & Berberidaceae & Chutro & Chutro & \begin{tabular}{|l} 
Indian \\
berbery
\end{tabular} & Stem & $\begin{array}{l}\text { Jaundice, fever, } \\
\text { skin disease }\end{array}$ \\
\hline $\begin{array}{l}\text { Bergenia ciliata (Ham) } \\
\text { sternb }\end{array}$ & Saxifragaceae & Pakhanbed & Pakhanbed & - & Nagikret & \\
\hline Bombax ceiba L & Bombacaceae & Kaga & simal & $\begin{array}{l}\text { Silk } \\
\text { cotton tree }\end{array}$ & Bark & \begin{tabular}{|l} 
Gum, \\
aphrodisiac, \\
diarrhoea, \\
dysentery \\
\end{tabular} \\
\hline Cannabis sativa $\mathrm{L}$ & Cannabaceae & Gaja & Gaja & - & Leaf & $\begin{array}{l}\text { Pain killer, } \\
\text { stomach ache }\end{array}$ \\
\hline Centella asiatica (L) Urb & Apiaceae & Chortapre & Ghortapre & $\begin{array}{l}\text { Water } \\
\text { pennywort }\end{array}$ & Stem & $\begin{array}{l}\text { Skin disease, } \\
\text { diuretic, } \\
\text { indigestion } \\
\end{array}$ \\
\hline Cumminum cyminum L & Apiaceae & Jera & Jera & Cumin & Seed & $\begin{array}{l}\text { Stomach ache, } \\
\text { dyspepsia, } \\
\text { diarrhoea }\end{array}$ \\
\hline Curcuma langa $\mathrm{L}$ & Zingiberaceae & Haledo & Haledo & - & Powder & $\begin{array}{l}\text { Cough, } \\
\text { bronchitis }\end{array}$ \\
\hline Cuscuta reflexa Roxb & Cuscutaceae & Urlara & Akash beli & & Tendril & Jaundice \\
\hline Dioscorea bulbifera L & Diascoreaceae & Gitta & Gitta & Air potato & Seed & $\begin{array}{l}\text { Piles, dysentery, } \\
\text { ulcer, }\end{array}$ \\
\hline $\begin{array}{l}\text { Eulaliopsis binata (Retz) } \\
\text { C.E. Hubbard }\end{array}$ & Poaceae & Babiyo & Babiyo & - & Root & ” \\
\hline Ficus racemosa & Moraceae & Lambrang & Dumri & - & Seed & \\
\hline Gamga pinnata Roxb & Burseraceae & Dab dabe & \begin{tabular}{|l|} 
Dab dabe \\
\end{tabular} & - & Bark & " \\
\hline $\begin{array}{l}\text { Girardiana diversifolia } \\
\text { (Lindl) Fris. }\end{array}$ & Urticaceae & Pachher & Allo & - & Bark & \\
\hline $\begin{array}{l}\text { Hedychium densiflorum } \\
\text { Wall }\end{array}$ & $\begin{array}{l}\text { Zingiberacea } \\
\mathrm{e}\end{array}$ & Besar & Besar & Turmeric & Powder & \\
\hline $\begin{array}{l}\text { Imperata cylindrical }(\mathrm{L}) \\
\text { Beauvois }\end{array}$ & " & Siru & Siru & - & Stem & Antihelmenthic \\
\hline Justicia adhatoda $\mathrm{L}$ & Acanthaceae & Ashuro & Ashuro & $\begin{array}{l}\text { Malabar } \\
\text { nut }\end{array}$ & Leaf & Medicine \\
\hline $\begin{array}{l}\text { Lalotropis gigantea }(\mathrm{L}) \\
\text { Dryand }\end{array}$ & \begin{tabular}{|l|} 
Asclepiadace \\
ae
\end{tabular} & Ank & Ank & $\begin{array}{l}\text { Giant } \\
\text { milk weed }\end{array}$ & Stem & " \\
\hline $\begin{array}{l}\text { Litsea cubeba (Lour) } \\
\text { pers. }\end{array}$ & Lauraceae & Prumo & Sil timur & - & Seed & Cholera \\
\hline $\begin{array}{l}\text { Litsea monopetala } \\
\text { (Roxb) Allen Ko Ster }\end{array}$ & " & Chabuth & Kutmero & - & Bark & \begin{tabular}{|l}
$\begin{array}{l}\text { Diarrhoea, } \\
\text { painkiller }\end{array}$ \\
\end{tabular} \\
\hline $\begin{array}{l}\text { Litsea salicifolia (Roxb. } \\
\text { ex. Nees) Hook }\end{array}$ & " & Harchut & Harchut & - & & Bark \\
\hline Melia azedarach (L) & Meliaceae & Bakaino & Bakaino & - & Seed & $\begin{array}{l}\text { Fever, } \\
\text { headache, loose } \\
\text { bowel }\end{array}$ \\
\hline Musa paradisiaca $\mathrm{L}$ & Musaceae & Moje & Kera & Banana & \begin{tabular}{|l}
$\begin{array}{l}\text { Leaf } \\
\text { extract }\end{array}$ \\
\end{tabular} & \begin{tabular}{|l|}
$\begin{array}{l}\text { Diarrhoea, } \\
\text { dysentery }\end{array}$ \\
\end{tabular} \\
\hline Ocimum basilicum $\mathrm{L}$ & Labiatae & Babari & Babari & - & Seed & $\begin{array}{l}\text { Gonorrhea, } \\
\text { diarrhoea, } \\
\text { dysentery }\end{array}$ \\
\hline Plumeria accuminata Air & Apocynaceae & Chuwa & Chuwa & \begin{tabular}{|l|} 
Pagoda \\
tree \\
\end{tabular} & \begin{tabular}{|l|} 
Plant \\
extract
\end{tabular} & " \\
\hline Potentilla fulgens wall & Rosaceae & Ghobarchhe & Bajradanti & - & Bark & Gum problem \\
\hline Premna intergrifolia L & Verbenaceae & Gindari & Gindari & - & Bark & ” \\
\hline
\end{tabular}


Our Nature (2003) 1: 37-41

\begin{tabular}{|l|l|l|l|l|l|l|}
\hline Psidium guajava Linn & Myrtaceae & Ambaru & Ambak & Guava & Bark & $\begin{array}{l}\text { Diarrhoea, } \\
\text { dysentery }\end{array}$ \\
\hline Schima wallichi Korth & Theaceae & Chillaune & Chillaune & - & Bark & Fever \\
\hline $\begin{array}{l}\text { Spondias pinnata (L.F.) } \\
\text { kurtz }\end{array}$ & $\begin{array}{l}\text { Anacardiacea } \\
\text { e }\end{array}$ & Chypang & Amaro & - & Seed & ” \\
\hline $\begin{array}{l}\text { Sterculia villosa } \text { Roxb. } \\
\text { ex. Smith }\end{array}$ & Sterculiaceae & Pat & Odal & Sterculia & Root & ” \\
\hline $\begin{array}{l}\text { Terminalia bellirica C.B. } \\
\text { Clarke }\end{array}$ & $\begin{array}{l}\text { Combretacea } \\
\text { e }\end{array}$ & Barro & Barro & $\begin{array}{l}\text { Belleric } \\
\text { myrobala } \\
\text { Terminalia chebula } \text { Retz }\end{array}$ & ” Seed & $\begin{array}{l}\text { Cough, } \\
\text { bronchitis }\end{array}$ \\
\hline $\begin{array}{l}\text { Thespesia lampas (cav) } \\
\text { Dalz \& Gibs }\end{array}$ & Malvaceae & Kapai & Ban Kapas & $\begin{array}{l}\text { Chebulic } \\
\text { myrobela }\end{array}$ & Seed & $\begin{array}{l}\text { Cough, } \\
\text { bronchitis }\end{array}$ \\
\hline $\begin{array}{l}\text { Tinospora cordifolia } \\
\text { Kurz }\end{array}$ & $\begin{array}{l}\text { Menispermac } \\
\text { eae }\end{array}$ & Gurju & Gurjo & Harro & Root & Jaundice \\
\hline
\end{tabular}

\section{Acknowledgements}

I express my sincere thanks to Dr. Nanda Bahadur Singh (Advisor and Research Guide, Central Dep. of Zoology, Kirtipur). I would like to express my deep sense of gratitude to Dr. R. S. L. Taylor (University of Calgary, Alberta, Canada) for invaluable suggestion. I'm also grateful to the people of Gorsyang VDC for their help in the study.

\section{References}

Anonymous 1984. Medicinal Plants of Nepal (Supplement Volume). Dept. Med. Plant. Kathmandu, Nepal.

Anonymous 2000. Nationalities of Nepal. Ministry of

Local Development, National Committee for Development of Nationalities.Kathmandu, Nepal.

Bangdel, L. S. and R. L. Fleming 1984. Nepalese Birds. Royal Nepal Academy

Bista, D. B. 1967. People of Nepal, HMG/N, KTM. Nepal.

Manandhar, N. P. 1991. Medicinal plant lore of Tamang tribe of Kabhrepalanchok District, Nepal. Economic Botany 45(1): 58-71.
Shrestha, J. 1994. Fishes of Nepal, Curriculum Development Center, Tribhuvan Univ. Nepal.

Shrestha, P. 1988. Contribution to the Ethnobotany of the Tamangs of Kathmandu Valley. Contribution to Nepalese Studies, 15(2): 147-266.

Shrestha, P. 1989.Ethnobotanical Observation on the Tamangs of Kathmandu Valley. In, Proc.of Nat.Confer. Sc. \& Tech. RONAST, Kathmandu, Apr. 24-29, 1988.pp:353-358.

Stainton, P. and O. Polunin 1994. Flowers of the Himalaya. Oxford University Press, India.

Shrestha, T. K. 1997. Mammals of Nepal. Mandela Book Point, Nepal.

Tamang, G. 1998. Ethnobiology of Tamang Tribe (unpublished thesis). M.Sc.Thesis. Tribhuvan Univ. Kirtipur, KTM. Nepal.

Taylor, R. S., N. P. Manandhar and G. H. N. Towers 1996 Antimicrobial and Antiviral Studies on Selected Plants of Nepal (Sherpa and Tamang People). Ethnobiology in Human Welfare. Ed. S. K. Jain. Deep Publication, New Delhi, pp 79-82. 